\title{
Selected statins produce rapid spinal motor neuron loss in vitro
}

\author{
Beth B Murinson ${ }^{1,2^{*}}$, Norman J Haughey ${ }^{1}$ and Nicholas J Maragakis ${ }^{1}$
}

\begin{abstract}
Background: Hmg-CoA reductase inhibitors (statins) are widely used to prevent disease associated with vascular disease and hyperlipidemia. Although side effects are uncommon, clinical observations suggest statin exposure may exacerbate neuromuscular diseases, including peripheral neuropathy and amyotrophic lateral sclerosis. Although some have postulated class-effects, prior studies of hepatocytes and myocytes indicate that the statins may exhibit differential effects. Studies of neuronal cells have been limited.

Methods: We examined the effects of statins on cultured neurons and Schwann cells. Cultured spinal motor neurons were grown on transwell inserts and assessed for viability using immunochemical staining for SMI-32. Cultured cortical neurons and Schwann cells were assessed using dynamic viability markers.

Results: 7 days of exposure to fluvastatin depleted spinal motor neurons in a dose-dependent manner with a $K_{D}$ of $<2 \mu \mathrm{M}$. Profound neurite loss was observed after 4 days exposure in culture. Other statins were found to produce toxic effects at much higher concentrations. In contrast, no such toxicity was observed for cultured Schwann cells or cortical neurons.

Conclusions: It is known from pharmacokinetic studies that daily treatment of young adults with fluvastatin can produce serum levels in the single micromolar range. We conclude that specific mechanisms may explain neuromuscular disease worsening with statins and further study is needed.
\end{abstract}

Keywords: ALS, Peripheral neuropathy, Statins, Toxicity, Motorneuronopathy

\section{Background}

Hmg-CoA reductase inhibitors (statins) are widely used for lowering cholesterol and the prevention of cardiovascular and cerebrovascular morbidity and mortality. Indeed, the use of statins has become more prevalent over time and selected populations, such as those discharged after hospital admission for stroke, have prevalence rates for statin prescription exceeding $80 \%$ [1].

Side effects of statin treatment are relatively uncommon. Rhabdomyolysis predominates as a side effect that may necessitate treatment cessation [2,3]. Myalgias are more prevalent but often accepted as part of treatment [4]. Laboratory studies to investigate the mechanisms underlying muscle-related effects have shown that statins have negative effects on oxidative phosphorylation by muscle mitochondria [5]. The concentrations at which

\footnotetext{
* Correspondence: bmurins1@jhmi.edu

${ }^{1}$ Department of Neurology, Johns Hopkins School of Medicine, Baltimore, USA

${ }^{2}$ Department of Neurology, Rambam Health Care Campus, Haifa, Israel
}

specific statins produce these effects varies widely and are generally considered to be outside the normal pharmacological dosing range except for cerivistatin (now withdrawn from the market) and possibly fluvastatin [6]. More recent studies have shown that statins alter important second messenger events, e.g., the linkage of proteins to membrane via lipophilic isoprenyl molecules [5] the synthesis of which is directly inhibited by statins [7].

Clinical observations have suggested that statin exposure may unmask or accelerate the course of amyotrophic lateral sclerosis, (ALS) [8]. Substantial controversy has accompanied these observations and based on current studies, it is not clear whether there is a causal association or merely selection bias $[9,10]$. Selection bias could arise if concerns about statins among ALS patients and their physicians lead to increased reporting of suspected effects. At present several questions remain unanswered. Among these is whether there is evidence that statins are directly toxic to spinal motor neurons. An additional
C Biomed Central

(c) 2012 Murinson et al.; licensee BioMed Central Ltd. This is an Open Access article distributed under the terms of the Creative Commons Attribution License (http://creativecommons.org/licenses/by/2.0), which permits unrestricted use, distribution, and reproduction in any medium, provided the original work is properly cited. 
unresolved question regards the role of statins with regards to peripheral neuropathy. Large scale epidemiological studies have suggested that statins are associated with an increased risk for peripheral neuropathy [11,12] but the magnitude and significance of this risk remains unresolved.

The culture of spinal motoneurons in organotypic spinal cord cultures has been an instructive methodology to better understand the neurobiology of the primary motor neurons [13] and has been used as a screening tool for the examination of potential candidate drugs for neuroprotective strategies in ALS [14]. Because of persistent concerns that statins may impact the course of ALS and prompt the development of peripheral neuropathy, we sought to examine the effects of statins on spinal motoneurons in culture.

\section{Methods}

\section{Spinal motoneuron cultures}

All reagents were obtained from Sigma-Aldrich unless otherwise specified. Organotypic spinal cord cultures were prepared under sterile conditions from the lumbar spinal cords of 8-day old rat pups, using an approved protocol as previously described [13]. In brief, transverse $350 \mathrm{~mm}$ sections were prepared with a McIlwain tissue chopper. Sections were suspended in sterile Gey's balanced salt solution (GIBCO) with glucose $(6.4 \mathrm{mg} / \mathrm{ml})$ and separated with gentle perturbation. Slices were transferred to Millipore trans-well inserts $(30 \mathrm{~mm}$ Millicell-CM, $0.4 \mathrm{~mm}$ pore membranes, Millipore, Bedford, MA). The transwell inserts were placed in $35 \mathrm{~mm}$ culture wells (Nalgene) containing $1 \mathrm{ml}$ of growth medium and cultured at $37^{\circ} \mathrm{C}$ in a humidified 5\%CO2/95\% air incubator (Forma Scientific, Marietta $\mathrm{OH}$ ). Growth medium consisted of Minimal essential medium-25 mM HEPES (50\% vol), heat-inactivated horse serum (25\% vol), and Hanks' balanced salt solution (Life Technologies, Rockville) (25\% vol), supplemented with D-glucose $(25.6 \mathrm{mg} / \mathrm{ml})$ and glutamine $(2 \mathrm{mM})$, final $\mathrm{pH}$ 7.2. Cultures were fed twice weekly for 14 days prior to treatment. Treatments were carried out for 7 days with a change of medium after 4 days . Statins (simvastatin (lactone) and fluvastatin from LKT Laboratories, St. Paul MN) were suspended in DMSO and diluted in growth medium before addition to culture. Control cultures contained DMSO at appropriate concentrations, in all cases less than $0.1 \%$.

\section{Cortical neuron cultures}

Cortical neuron cultures were prepared as previously described from E18 rats [15]. Cultures were plated on poly-D-lysine coated plates at a density of 106 cells $/ \mathrm{ml}$, and established for 2 weeks prior to experimental assessment. Plating medium consisted of minimal essential medium containing $10 \%$ fetal bovine serum and antibiotics
(1\% of penicillin G $104 \mathrm{U} / \mathrm{ml}$, streptomycin $10 \mathrm{mg} / \mathrm{ml}$, amphotericin B $25 \mathrm{ug} / \mathrm{ml}$ ). Three hours after plating, medium was changed to culture medium consisting of Neurobasal medium with 1x B-27 supplement (Life Technologies, Rockville, MD).

\section{Schwann cell cultures}

Schwann cell cultures were prepared as previously described from 1-day-old rat pups[16,17]. Cultures were established for 2 weeks prior to experimental assessment. Culture medium consisted of Neurobasal medium (GIBCO) with $1 \%$ fetal bovine serum (Hyclone, Logan UT) and glial cell line-derived neurotrophic factor (GDNF, ) at $10 \mathrm{ug} / \mathrm{ml}$. Schwann cells were plated into 96-well plates, using brief trypsinization, $24 \mathrm{~h}$ prior to experimental treatment.

\section{Immunochemistry and assessment of spinal motoneuron cultures}

Cultures were fixed with $4 \%$ paraformaldehyde in $0.1 \mathrm{M}$ Sorenson's buffered solution ( $\mathrm{pH}$ 7.4) for $30 \mathrm{~min}$ at room temperature. Immunochemistry was performed as previously described [18]. Slices were permeabilized with methanol, $20 \mathrm{~min}$ at $4^{\circ} \mathrm{C}$. Rinsing with Tris-buffered saline preceded blocking with $10 \%$ normal goat serum, $1 \mathrm{~h}$ at room temperature. Exposure to primary antibody (SMI$32,1: 8000)$ was at $4^{\circ} \mathrm{C}$ overnight. Signal amplification with Vectastain ABC (Vector, Burlingame, CA) preceded chromogenic development with diamino-benzidine (DAB) (Polyscience, Warrington, PA).

Spinal motor neurons were specifically identified in the organotypic slices based on cell size, morphology, location in the ventral horn, and dense staining with SMI-32. Motor neuron counts were carried out in a blinded fashion on 12-16 explants for each condition and confirmed by a second investigator.

Additional cultures and immunochemical studies were performed on organotypic slice cultures with 4 days of treatment to ascertain whether neuritic degeneration preceded motoneuron degeneration. For this, slices were placed onto collagen-coated transwell inserts, known to promote the outgrowth of neurites under appropriate conditions. Chromogenic development was performed using FITC-conjugated secondary antibody. Visualization of these slices was carried out using a Nikon Fluorescence microscope.

\section{Assessment of cortical neuron cultures and Schwann cell cultures}

As a measure of statin cytotoxicity for cortical neurons and Schwann cells, ATP levels were measured as previously described [19] with minor modifications. Briefly, cells were plated into 96 well plates for $24 \mathrm{~h}$ prior to treatment with statins, vehicle control solution or medium 
control solution. Following $24 \mathrm{~h}$ of experimental treatment, measurement of ATP levels was performed using a commercially available luciferase-linked ATPase enzymatic assay (Vialight Plus, Cambrex).

\section{Statistical analysis}

The number of spinal motor neurons in the slices of the experimental groups did not follow a normal distribution, for this reason median values are reported. For between group comparisons, data were transformed using a logarithmic function $(\log (\mathrm{N}+1))$. Comparisons between groups were made on transformed data using a Chi-squared test with correction for multiple comparisons. 50\% toxicity value for fluvastatin was estimated from data following a log-linear transformation of the data.

\section{Results}

Three commonly used statins were tested for toxicity to spinal motoneurons in culture, these included fluvastatin, pravastatin, and simvastatin. As a group, the organotypic slice cultures retained normal conformational properties and were adherent to the membrane indicative of overall cellular integrity, consistent with observations of little or no toxicity of statins for glial cells. Spinal motor neurons were strikingly depleted or absent from cultures exposed to fluvastatin, compare Figure 1A and $1 \mathrm{~B}$.

Quantitation of spinal motoneurons showed that the median number per slice was 15 in the vehicle control group. The median number of motoneurons per slice was 0 in the $200 \mu \mathrm{M}$ fluvastatin and simvastatin treatment groups, a significant decrease in motoneuron numbers $(p<.001)$, Figure $1 \mathrm{C}$. Treatment with $200 \mu \mathrm{M}$ pravastatin yielded a median of 1 cell per slice, and also showed toxicity compared with control $(p<.001)$. A limited dose-response curve for fluvastatin was obtained. Fluvastatin at $20 \mu \mathrm{M}$, was toxic for cultured motoneurons, median cells per slice was $0,(p<.001)$. Fluvastatin at $2 \mu \mathrm{M}$, was associated with partial toxicity, the median number of motoneurons of 5 . This was significantly different from control, $(p<.001)$ and from $20 \mu \mathrm{M}$ and $200 \mu \mathrm{M}$ fluvastatin, $(p<.001)$. Replicate experiments showed analogous results.

Further assessment was made using cultures with only 4 days of statin exposure. Control organotypic slice cultures grown on collagen coated membranes exhibited robust ventral neurite outgrowth after 2.5 weeks in culture. By contrast, organotypic slices exposed to $200 \mu \mathrm{M}$ fluvastatin for $96 \mathrm{~h}$ demonstrated profound neuritic degeneration, Figure 2. Motoneuron staining, somewhat reduced, was still observable in these cultures.

In order to assess whether spinal motor neurons were unusual in exhibiting cytotoxic responses to statin exposure, we assessed the effects of statin treatment on

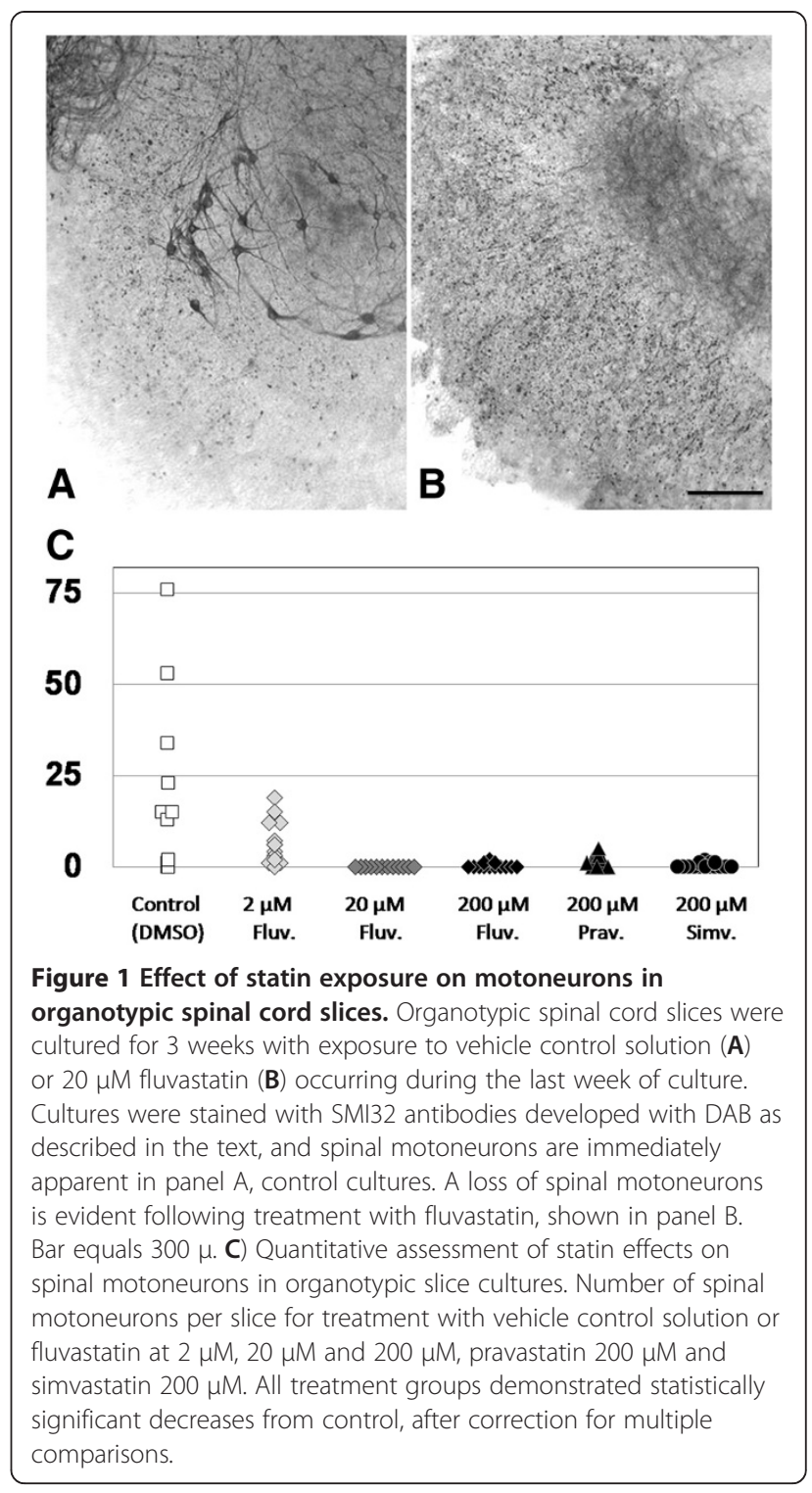

cortical neuron cultures and primary cultures of Schwann cells. No potentially relevant cytotoxic effects were observed for these cell types, Figure 3.

\section{Discussion}

In this study, we demonstrate that in vitro exposure of spinal motoneurons to selected statins, especially fluvastatin, resulted in marked cell loss. Shorter term exposures to fluvastatin resulted in neuritic degeneration. Other statins, specifically pravastatin and simvastatin, also exhibited toxic effects towards spinal motor neurons, but at much higher concentrations. The susceptibility of spinal motor neurons was in contrast to primary cultures of cortical neurons and peripheral nerve-derived Schwann cells which did not demonstrate significant deleterious changes. The results of this study provide evidence that specific 


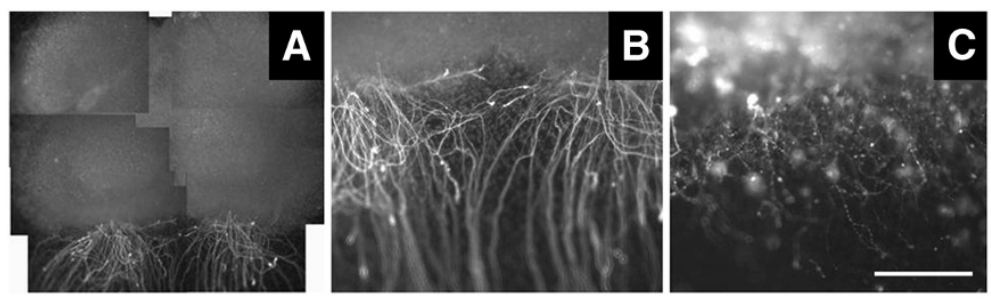

Figure 2 Effect of statin exposure on neurite outgrowth. Fluvastatin exposure for $96 \mathrm{~h}$ resulted in a marked degeneration of neurites growing out from organotypic spinal cord cultures, when plated on collagen coated membranes. Cultures were stained with SMI32 antibodies developed with FITC-conjugated secondary antibody as described in the text. Vigorous outgrowth of ventral horn neurites is apparent in panel $\mathbf{A}$, montage view (bar equals $1 \mathrm{~mm}$ ) and panel $\mathbf{B}$, detail view (bar equals $500 \mu \mathrm{m}$ ). Neuritic degeneration is seen in panel C (bar equals $500 \mu \mathrm{m}$ ).

pathophysiological mechanisms may underlie reports of neuromuscular disease exacerbation with statin exposure, however the clinical implications of this study remain to be determined.

There are multiple factors which might account for the differences between the clear in vitro toxicity reported here and the uncertainty surrounding observations of statin toxicity for spinal motor systems in clinical settings. Among the biological factors proposed to explain the wide clinical tolerability of statins are pharmacokinetics. Statins as a group are subject to high first-pass metabolism by the liver. This means that systemic levels are substantially below what might be predicted based on simple dilutional calculations. Statins do vary in the degree of first pass metabolism with the earlier-developed statins being more extensively metabolized [6]. Interestingly the low first-pass metabolism of cerivistatin, initially hailed as a breakthrough, may have contributed to the high-rate of serious adverse events [3]. In addition, most statins are hydrophobic, with several newer statins being especially so. Pravastatin is uniquely hydrophilic and likely requires
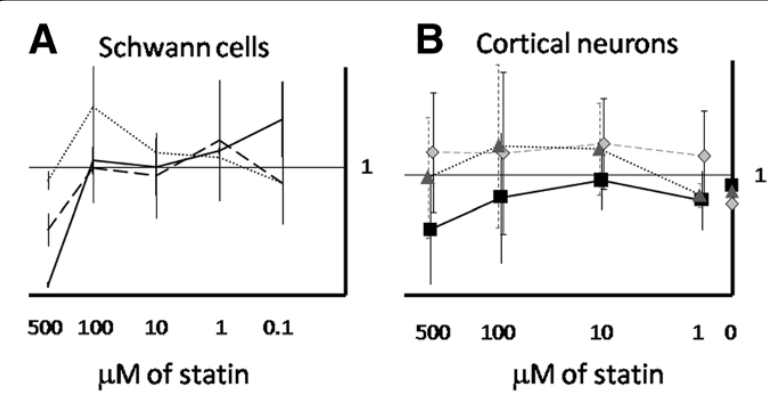

Figure 3 Effect of statin exposure on primary Schwann cell and cortical neuron cultures. A) Statin treatment of Schwann cells for $24 \mathrm{~h}$ did not result in significant toxicity except for very high concentrations of fluvastatin and simvastatin $(500 \mu \mathrm{M})$. Three different statins are shown: fluvastatin, solid line; simvastatin, long dash; pravastatin, short dash. B) Statin treatment of cortical neurons for $24 \mathrm{~h}$ did not result in significant toxicity. Three different statins are shown: fluvastatin, solid line; lovastatin, long dash; pravastatin, short dash. Data are shown normalized to vehicle control, error bars indicate standard deviation from the mean. distinct consideration when considering systemic effects. In our study, there was the suggestion that pravastatin may be somewhat less toxic to cultured spinal motoneurons that fluvastatin, however further studies are needed. Finally, compartmental pharmacokinetics may limit the effects of statins on spinal motor neurons. The extent to which specific statins attain significant concentrations in the spinal fluid is not well understood. Studies in laboratory animals have indicated that the permeability of lipophilic statins, simvastatin and lovastatin into rat brain is orders of magnitude greater than that of pravastatin [20]. Thus, there are multiple pharmacokinetic reasons why statins may exhibit lower in vivo than in vitro toxicity.

Pharmacokinetic data for fluvastatin from clinical trials of the drug, suggest that a more cautious interpretation may warranted. From the early pharmacokinetic studies in man, it is estimated that the peak daily serum levels of fluvastatin after a $40 \mathrm{mg}$ dose is in the low single micromolar range [21]. The results of the fluvastatin dose response studies we report here provide evidence that the drug concentration necessary to produce $50 \%$ toxicity for spinal motoneurons in culture is less than $1 \mu \mathrm{M}$. Taken together, these data suggest that systemic levels of statins may approach levels that are toxic to spinal motoneurons and that particular mechanisms must account for the relatively infrequent occurrence of clinically demonstrable toxicity. Perhaps sequestration of spinal motoneurons in the spinal space is one such mechanism.

The pharmacokinetics of fluvastatinare are such that peak concentrations are present in the body for only a short period of time following single-dose administration [21]. For this reason, early fluvastatin treatment followed a twice-daily dosing regimen. This approach has subsequently been supplanted by the use of extended release formulations but for reasons that are not known, fluvastatin remains among the least commonly prescribed statins.

Based on these studies, it is not possible to specify a mechanism or mechanisms by which statins produce depletion of spinal motoneurons in culture. Intriguingly, somewhat analogous effects of statins have been reported in myotube cultures, where it was 
observed that fluvastatin and simvastatin exhibit clearly evident toxicity, the effects of pravastatin were apparently more mild [6]. Mechanisms by which statins have produced undesirable and desirable effects in other systems continue be explored. The effects of statins are widely acknowledged to be protean. Statins have profound impacts on inflammatory signaling pathways [22,23], are known to effect the linkage of $G$ proteins to the various cell membranes [24,25], are thought to play a role in cancer signaling [26], have been shown to have direct actions on carnitine palmitoyl transferase localized to mitochondrial membranes [6], can disrupt oxidative phosphorylation and mitochondrial membrane potential [6] as well as interfering with cholesterol synthesis [27]. As an example of a one potential mechanism, statins have been shown to disrupt the attachment of Rho and Ras-family G proteins to cell membranes. It is interesting that one Ras-family protein, Rab5, is critically important for early neuronal endocytosis [28] and has been implicated in producing an ALS phenotype resulting from Alsin mutations [29]. The se animals may represent an especially attractive model system for further exploration of statin effects on motor neurons. Among these studies is a report that statins promote apoptosis of a glioma cell line mediated by ERK1/2 and AKT [25]. This is in contrast to our observations of robust resistance to statin effects in primary cultures of Schwann cells suggesting that important differences between normal and transformed glia that underlie statin effects on glioma cells. Thus, statins have a variety of effects on cell signaling. We here describe the observation of deleterious effects on the survival of spinal motor neurons in culture and because the mechanisms of statin effects on specific neuronal cell populations remain relatively obscure, additional study is warranted.

\section{Conclusions}

In this study, we demonstrate that in vitro exposure of spinal motoneurons to selected statins, especially fluvastatin, resulted in marked cell loss. Shorter term exposures to fluvastatin resulted in neuritic degeneration. The results of this study provide evidence that specific pathophysiological mechanisms may underlie reports of neuromuscular disease exacerbation with statin exposure.

\section{Competing interests}

The authors declare that they have no competing interests.

\section{Authors' contributions}

BBM contributed to the planning and conduct of all the experiments, statistical analysis, manuscript preparation and revisions. NH contributed to the planning of the experiments, the preparation of the cortical neuronal cultures and manuscript revisions. NM contributed to the planning of the experiments, the preparation and interpretation of the spinal motor neuron cultures and manuscript revisions. All authors read and approved the final manuscript.

\section{Acknowledgements}

The authors thank Carol Coccia for expert technical assistance, Dr. Ahmet Hoke for the gift of $50 \mathrm{~B} 11$ cells, and Drs. John Griffin and Thomas Brushart for discussions of this work. This work was supported by a Career

Development Award from the National Institutes of Health (K08NS048146) to Dr. Murinson and preparation of the manuscript was supported in part by the Legacy Heritage Fund. Dr Maragakis is supported in part by a grant from the Packard Center for ALS Research.

Received: 18 October 2011 Accepted: 20 February 2012

Published: 15 June 2012

\section{References}

1. Ovbiagele B, Schwamm LH, Smith EE, Hernandez AF, Olson DM, Pan W, Fonarow GC, Saver JL: Recent nationwide trends in discharge statin treatment of hospitalized patients with stroke. Stroke 2010, 41:1508-1513.

2. Graham DJ, Staffa JA, Shatin D, Andrade SE, Schech SD, La GL, Gurwitz JH, Chan KA, Goodman MJ, Platt R: Incidence of hospitalized rhabdomyolysis in patients treated with lipid-lowering drugs. JAMA 2004, 292:2585-2590.

3. Staffa JA, Chang J, Green L: Cerivastatin and reports of fatal rhabdomyolysis. N Engl J Med 2002, 346:539-540.

4. Thompson PD, Clarkson P, Karas RH: Statin-associated myopathy. JAMA 2003, 289:1681-1690

5. Johnson TE, Zhang X, Bleicher KB, Dysart G, Loughlin AF, Schaefer WH, Umbenhauer DR: Statins induce apoptosis in rat and human myotube cultures by inhibiting protein geranylgeranylation but not ubiquinone. Toxicol Appl Pharmacol 2004, 200:237-250.

6. Kaufmann P, Torok M, Zahno A, Waldhauser KM, Brecht K, Krahenbuhl S: Toxicity of statins on rat skeletal muscle mitochondria. Cell Mol Life Sci 2006, 63:2415-2425.

7. Matzno S, Yasuda S, Juman S, Yamamoto Y, Nagareya-Ishida N, Tazuya-Murayama K, Nakabayashi T, Matsuyama K: Statin-induced apoptosis linked with membrane farnesylated Ras small G protein depletion, rather than geranylated Rho protein. J Pharm Pharmacol 2005, 57:1475-1484.

8. Zinman L, Sadeghi R, Gawel M, Patton D, Kiss A: Are statin medications safe in patients with ALS? Amyotroph Lateral Scler 2008, 9:223-228.

9. Drory VE, Bronipolsky T, Artamonov I, Nefussy B: Influence of statins treatment on survival in patients with amyotrophic lateral sclerosis. J Neurol Sci 2008, 273:81-83.

10. Edwards IR, Star K, Kiuru A: Statins, neuromuscular degenerative disease and an amyotrophic lateral sclerosis-like syndrome: an analysis of individual case safety reports from vigibase. Drug Saf 2007, 30:515-525.

11. Corrao G, Zambon A, Bertù L, Botteri E, Leoni O, Contiero P: Lipid lowering drugs prescription and the risk of peripheral neuropathy: an exploratory case-control study using automated databases. J Epidemiol Community Health, 58(12):1047-1051. 12-1-2004.

12. Gaist D, Jeppesen U, Andersen M, Garcia Rodrigeuz LA, Hallas J, Sindrup SH: Statins and risk of polyneuropathy: a case-control study. Neurology 2002, 273:81-83.

13. Rothstein JD, Jin L, Dykes-Hoberg M, Kund RW: Chronic inhibition of glutamate uptake produces a model of slow neurotoxicity. Proc Natl Acad Sci U S A 1993, 90:6591-6595.

14. Rothstein JD, Patel S, Regan MR, Haenggeli C, Huang YH, Bergles DE, Jin L, Dykes HM, Vidensky S, Chung DS, Toan SV, Bruijn LI, Su ZZ, Gupta P, Fisher PB: Beta-lactam antibiotics offer neuroprotection by increasing glutamate transporter expression. Nature 2005, 433:73-77.

15. Szklarczyk A, Ewaleifoh O, Beique JC, Wang Y, Knorr D, Haughey N, Malpica T, Mattson MP, Huganir R, Conant K: MMP-7 cleaves the NR1 NMDA receptor subunit and modifies NMDA receptor function. FASEB J 2008, 22:3757-3767

16. Brockes JP, Fields KL, Raff MC: Studies on cultured rat Schwann cells. I. Establishment of purified populations from cultures of peripheral nerve. Brain Res 1979, 165:105-118.

17. Keswani SC, Polley M, Pardo CA, Griffin JW, McArthur JC, Hoke A: Schwann cell chemokine receptors mediate HIV-1 gp120 toxicity to sensory neurons. Ann Neurol 2003, 54:287-296.

18. Maragakis NJ, Rao MS, Llado J, Wong V, Xue H, Pardo A, Herring J, Kerr D, Coccia C, Rothstein JD: Glial restricted precursors protect against chronic glutamate neurotoxicity of motor neurons in vitro. GLIA 2005, 50:145-159. 
19. Chen W, Mi R, Haughey N, Oz M, Hoke A: Immortalization and characterization of a nociceptive dorsal root ganglion sensory neuronal line. J Peripher Nerv Syst 2007, 12:121-130.

20. Saheki A, Terasaki T, Tamai I, Tsuji A: In vivo and in vitro blood-brain barrier transport of 3-hydroxy-3-methylglutaryl coenzyme A (HMG-CoA) reductase inhibitors. Pharm Res 1994, 11:305-311.

21. Deslypere JP: Clinical implications of the biopharmaceutical properties of fluvastatin. Am J Cardiol 1994, 73:12D-17D.

22. Jain MK, Ridker PM: Anti-inflammatory effects of statins: clinical evidence and basic mechanisms. Nat Rev Drug Discov 2005, 4:977-987.

23. Youssef S, Stuve O, Patarroyo JC, Ruiz PJ, Radosevich JL, Hur EM, Bravo M, Mitchell DJ, Sobel RA, Steinman L, Zamvil SS: The HMG-CoA reductase inhibitor, atorvastatin, promotes a Th2 bias and reverses paralysis in central nervous system autoimmune disease. Nature 2002, 420:78-84.

24. Araki M, Maeda M, Motojima K: Hydrophobic statins induce autophagy and cell death in human rhabdomyosarcoma cells by depleting geranylgeranyl diphosphate. Eur J Pharmacol 2012, 674:95-103.

25. Yanae M, Tsubaki M, Satou T, Itoh T, Imano M, Yamazoe Y, Nishida S: Statin-induced apoptosis via the suppression of ERK1/2 and Akt activation by inhibition of the geranylgeranyl-pyrophosphate biosynthesis in glioblastoma. J Exp Clin Cancer Res 2011, 30:74.

26. Mohammed A, Qian L, Janakiram NB, Lightfoot S, Steele VE, Rao CV: Atorvastatin delays progression of pancreatic lesions to carcinoma by regulating $\mathrm{PI} 3 / \mathrm{AKT}$ signaling in $\mathrm{p} 48(\mathrm{Cre} /+)$. LSL-Kras(G12D/+) mice. Int J Cancer 2012,

27. Goldstein JL, Brown MS: Regulation of the mevalonate pathway. Nature 1990, 343:425-430

28. Tall GG, Barbieri MA, Stahl PD, Horazdovsky BF: Ras-activated endocytosis is mediated by the Rab5 guanine nucleotide exchange activity of RIN1. Dev Cell 2001, 1:73-82.

29. Hadano S, Benn SC, Kakuta S, Otomo A, Sudo K, Kunita R, Suzuki-Utsunomiya K, Mizumura H, Shefner JM, Cox GA, Iwakura Y, Brown RH Jr, Ikeda JE: Mice deficient in the Rab5 guanine nucleotide exchange factor ALS2/alsin exhibit age-dependent neurological deficits and altered endosome trafficking. Hum Mol Genet 2006, 15:233-250.

doi:10.1186/1471-2474-13-100

Cite this article as: Murinson et al:: Selected statins produce rapid spinal motor neuron loss in vitro. BMC Musculoskeletal Disorders 2012 13:100.

\section{Submit your next manuscript to BioMed Central and take full advantage of:}

- Convenient online submission

- Thorough peer review

- No space constraints or color figure charges

- Immediate publication on acceptance

- Inclusion in PubMed, CAS, Scopus and Google Scholar

- Research which is freely available for redistribution 